\title{
Narração de histórias por crianças com distúrbio específico de linguagem $* * * *$
}

\author{
Narration of stories by children with specific language impairment
}

\author{
Débora Maria Befi-Lopes* \\ Ana Carolina Paiva Bento** \\ Jacy Perissinoto***
}

\section{*Fonoaudióloga. Professora Associada Livre Docente do Curso de Fonoaudiologia do Departamento de Fisioterapia, Fonoaudiologia e Terapia Ocupacional da Faculdade de Medicina da Universidade de São Paulo - Área de Linguagem Infantil. Endereço para correspondência: \\ Rua Cipotânea, 51 - Campus Cidade Universitária - São Paulo - SP - CEP 05360-160 \\ (dmblopes@usp.br)}

**Fonoaudióloga. Mestranda do Programa de Pós-Graduação em Ciências da Reabilitação - Área Comunicação Humana da Faculdade de Medicina da Universidade de São Paulo - Departamento de Fisioterapia, Fonoaudiologia e Terapia Ocupacional da Faculdade de Medicina da Universidade de São Paulo - Área de Linguagem Infantil.

***Fonoaudióloga. Doutora em Distúrbios da Comunicação Humana pela Universidade Federal de São Paulo. Professora Adjunta do Curso de Fonoaudiologia da Universidade de São Paulo.

****Trabalho Realizado no Departamento de Fonoaudiologia, Fisioterapia e Terapia Ocupacional da Faculdade de Medicina da Universidade de São Paulo - Área de Linguagem Infantil.

Artigo Original de Pesquisa

Artigo Submetido a Avaliação por Pares

Conflito de Interesse: não

Recebido em 13.11.2007.

Revisado em 10.03.2008; 16.05.2008.

Aceito para Publicação em 16.05.2008.

\section{Abstract}

Background: narrative abilities provide rich information about the linguistic, cognitive and social competences of children with typical language development and with SLI (Specific Language Impairment). Children with SLI present deficits in speech elaboration, which is generally confusing and repetitive. Furthermore, there may be difficulty with text organization, understanding the underlying time and cause-effect relationships and in the development of the structural knowledge needed for comprehension. Aim: to characterize the narration of stories by children with SLI regarding the type and content of speech and to compare their performance to that of their typically developing peers, matched according to the chronological age. Method: two groups participated in this study: Control Group (CG), 24 children with no language deficits and Research Group (RG), 8 children with the diagnosis of SLI. To elicit the narratives, a series of 15 stories were used, represented by illustrations containing four scenes each. These sequences were created and classified as mechanical, behavioral and intentional, according to the relationship established between the characters. Results: children with SLI presented poorer narratives when compared to their typically developing peers, independent of the type of story which was presented. Moreover, children with SLI showed a similar perception of the mental states when compared to children with normal development. Conclusions: these results indicate that, regardless the type of story, children with SLI have difficulties in the use of language, that is, with the linguistic abilities necessary to narrate stories and not in the perception of the characters' mental state.

Key Words: Language; Language Development Disorders; Narration.

\section{Resumo}

Tema: as habilidades narrativas podem fornecer ricas informações sobre as competências lingüísticas, cognitivas e sociais das crianças com desenvolvimento típico e com DEL (Distúrbio Específico de Linguagem). Crianças com DEL apresentam déficits na elaboração do discurso, que geralmente são confusos e repetitivos. Além disso, há dificuldades na organização textual, compreensão da temporalidade, relações de causa e efeito e desenvolvimento de conhecimento estrutural necessária para a compreensão da informação. Objetivo: caracterizar a narração de histórias por crianças com DEL com relação ao tipo e conteúdo do discurso e comparar o desempenho destes sujeitos com seus pares cronológicos em desenvolvimento típico. Método: participaram deste estudo dois grupos: Grupo Controle (GC), sem alterações de linguagem, composto por 24 sujeitos e Grupo Pesquisa (GP), com diagnóstico de DEL, composto por 8 sujeitos. Para eliciar as narrativas foi utilizada uma série de 15 histórias, representadas por figuras, compostas por quatro cenas cada. Essas seqüências foram criadas e classificadas em mecânicas, comportamentais e intencionais, segundo as relações envolvidas entre as personagens. Resultados: As crianças com DEL apresentam narrativas mais rudimentares se compararmos a seus pares cronológicos com desenvolvimento típico de linguagem independente do tipo de história fornecida. Além disso, as crianças com DEL apresentaram percepção dos estados mentais semelhante às crianças com desenvolvimento normal. Conclusões: estes resultados indicam que, independente do tipo de história fornecida, a dificuldade destas crianças está na utilização da língua, ou seja, nas habilidades lingüísticas necessárias na narração de histórias e não na percepção dos estados mentais dos personagens. Palavras-Chave: Linguagem; Transtornos do Desenvolvimento da Linguagem; Narrativa. 


\section{Introdução}

As manifestações de linguagem encontradas no Distúrbio Específico de Linguagem (DEL) são variadas, estando na dependência do grau de gravidade do quadro, podendo ser mutáveis durante o desenvolvimento. Geralmente as manifestações são: simplificações fonológicas, freqüentemente desviantes (simplificações não observadas no processo normal de aquisição de linguagem); vocabulário restrito, com uso demasiado de dêiticos, perífrases e gestos representativos; dificuldade em adquirir novas palavras; estruturação gramatical simplificada e pouco variada; ordenação de palavras de forma não usual. Quando a compreensão está comprometida, observam-se dificuldades em entender sentenças ou palavras específicas como marcadores espaciais ou temporais, realização de comandos lingüísticos de forma incorreta, respostas incorretas sob questionamento e dificuldade em manter o tópico de conversação pelas dificuldades de compreensão ${ }^{(1-4)}$.

Sendo o DEL uma entidade nosológica complexa há muito a ser investigado. Do ponto de vista das manifestações da linguagem, é fundamental identificar as possíveis falhas nos diferentes processos psicolingüísticos e de processamento da linguagem oral e escrita, a fim de tornar o processo terapêutico melhor direcionado ${ }^{(5)}$.

Dessa forma, as habilidades narrativas podem nos fornecer ricas e variadas informações sobre as competências lingüísticas, cognitivas e sociais das crianças com desenvolvimento típico e com DEL ${ }^{(6)}$.

A narrativa é uma tarefa complexa que requer integração lingüística, cognitiva e habilidades sociais, e seu potencial como avaliação clínica tem sido explorado recentemente ${ }^{(7)}$. Além disso, a narrativa oferece um método para se investigar questões teóricas acerca da relação entre a linguagem e a cognição ${ }^{(8)}$.

Estudos observaram que as crianças com DEL, além das dificuldades que já foram citadas, apresentam também déficits na elaboração do discurso, que geralmente são confusos e repetitivos ${ }^{(9-10)}$.

Westby et al. ${ }^{(11)}$ referem que crianças com DEL apresentam dificuldades na construção do que o ouvinte necessita ouvir para compreender a mensagem e conseqüentemente acabam por realizar inferências inadequadas. Além disso, há dificuldades na organização textual, compreensão da temporalidade, relações de causa e efeito e desenvolvimento de conhecimento estrutural necessária para a compreensão da informação. Sendo assim, os objetivos do presente estudo são caracterizar a narração de histórias por crianças com DEL com relação ao tipo e conteúdo do discurso e comparar o desempenho destes sujeitos com seus pares cronológicos em desenvolvimento típico.

\section{Método}

Participaram deste estudo dois grupos de crianças: Grupo Controle (GC) e Grupo Pesquisa (GP), na proporção de 3-1 (controle-pesquisa). Todos os responsáveis assinaram o termo de consentimento livre e esclarecido que compõe o projeto aprovado pela Comissão de Ética para Análise de Projetos de Pesquisa - CAPPesq da Diretoria Clínica do Hospital das Clínicas e da Faculdade de Medicina da Universidade de São Paulo, sob número 0666/07.

O GC foi composto por 24 crianças, de ambos os sexos, com faixa etária média de 8:8 anos (7:2 e 10:10 anos), pertencentes a uma Escola Estadual da região leste da cidade de São Paulo.

Os critérios de seleção para o GC foram: ausência de queixa ou tratamento fonoaudiológico anterior, bom padrão comunicativo e desempenho escolar satisfatório segundo as professoras. Além disso, deveriam apresentar desempenho adequado em prova de fonologia e em tarefas que envolviam consciência fonológica e leitura e escrita ${ }^{(12-13)}$.

O GP foi composto por oito crianças com diagnóstico de DEL de ambos os sexos, com faixa etária média de 8:9 anos (7:0 e 10:6 anos), que freqüentavam terapia fonoaudiológica no Laboratório de Investigação Fonoaudiológica em Desenvolvimento da Linguagem e suas Alterações (LIF-ADL) do Departamento de Fisioterapia, Fonoaudiologia e Terapia Ocupacional da Faculdade de Medicina da Universidade de São Paulo (FMUSP).

Para eliciar as narrativas foi utilizada uma série de 15 histórias, representadas por figuras, compostas por quatro cenas cada. Baron-Cohen et al. ${ }^{(14)}$ criaram e classificaram as seqüências como mecânicas, comportamentais e intencionais, segundo as relações envolvidas entre as personagens. 
Mecânica (SM)

Objetos ou objetos e pessoas interagindo casualmente, uns com os outros.

Uma das seqüências apresenta um ovo em cima de uma mesa, o ovo rola sobre a mesa, cai da mesa e quebra ao cair no chão.

São utilizadas seis seqüências mecânicas sendo que três delas envolvem somente objetos e as demais envolvem tanto objetos como pessoas.

\section{Comportamental (SC)}

Uma ou mais pessoas atuando em situações rotineiras, que não requerem atribuição de estados mentais.

Em uma das seqüências temos um homem que está preparando a massa de uma torta, ele a coloca numa forma, coloca a forma no forno e em seguida a torta fica pronta.

São utilizadas seis seqüências comportamentais.

Intencional (SI)

Pessoas atuando em atividades diárias que requerem atribuição de estados mentais.

Em uma das seqüências temos um menino com uma caixa de bombons, ele coloca os bombons sobre a mesa e sai para jogar bola com os amigos. Enquanto isso, sua mãe come todos os bombons e quando ele volta para comer os bombons ele fica chateado ao ver que a caixa de bombons está vazia.

São utilizadas três sequiências intencionais.

Perissinoto ${ }^{(15)}$, a partir das classificações descritas acima, elaborou as 15 histórias, bem como sua representação em desenhos que foram utilizados no presente estudo.

A pesquisadora explicou aos participantes que as sequiências de figuras formavam uma história. A primeira cena de cada história era mostrada para a criança, a avaliadora perguntava o que ela estava vendo na figura. Caso a criança não soubesse nomear todos os elementos da primeira cena a avaliadora explicava e somente após a compreensão de todos os elementos as outras três figuras eram fornecidas à criança para que pudesse organizá-las e então era solicitado que narrasse à história formada.

As crianças do GP e GC narraram todas as histórias (gravadas digitalmente) e então, estas foram transcritas e analisadas pela pesquisadora.

Os resultados foram analisados segundo os critérios definidos por Baron-Cohen et al. ${ }^{(14)}$ :
Tipo de discurso:

. descritivo sem conectivos - não há presença de conectivos que estabeleçam uma relação seqüencial entre as cenas;

. descritivo com conectivos - o uso de conectivos estabelece uma relação aditiva entre as cenas, sem fazer relação direta entre os acontecimentos narrados em uma cena e na subseqüente;

. causal implícito - uso de expressões que não as determinantes diretas de causa, mas que expressam relação de causalidade;

. causal explícito - uso de conjunções causais ou explicitação do agente causador;

. intencional implícito - uso de interjeições ou expressões não-lingüísticas que expressem desejo ou sentimento da personagem;

. intencional explícito - há a expressão de estados mentais das personagens, através da atribuição ou do uso de verbos que expressam desejo, ou quando a fala da personagem é narrada na forma de discurso direto.

Caso ocorresse em uma mesma narrativa mais de um tipo de discurso, a classificação foi feita de acordo com o tipo de narrativa mais complexa.

Conteúdo do Discurso ${ }^{(16)}$ :

. pertinência - se o discurso foi referente ao tema da seqüência;

. coerência - se o discurso continha começo, meio e fim, apresentando estrutura semântica completa. Esse critério está prioritariamente relacionado com a compreensão da narrativa pelo interlocutor; . completude - se todas as ações representadas nas cenas foram narradas, independente da ordem em que ocorreram ou da compreensão dos acontecimentos.

\section{Resultados}

Para a análise estatística dos dados foi utilizado o teste não paramétrico Qui-quadrado (X2) e o nível de significância adotado foi de 5\%. As respostas estatisticamente significantes estão marcadas com um asterisco e em negrito.

Os grupos se diferenciaram quanto ao tipo de narrativa ou discurso $(\mathrm{X} 2=124,47 ; \mathrm{p}<0,001 *)$.

O GP produziu $19 \%$ de narrativas descritivas sem conectivo; $40,5 \%$ de narrativas descritivas com conectivo, 30,2\% de narrativas causais implícitas, $1,7 \%$ de narrativas causais explícitas, 3,4\% de narrativas intencionais implícitas e 5,2\% de narrativas intencionais explícitas. 
Já o GC produziu $14,4 \%$ de narrativas descritivas com conectivo, $48,9 \%$ de narrativas causais implícitas, 5\% de narrativas causais explícitas, $7,8 \%$ de narrativas intencionais implícitas, $23,9 \%$ de narrativas intencionais explícitas e não apresentou nenhuma narrativa descritiva sem conectivo.

Portanto, para o GP há um predomínio das narrativas descritivas com conectivos, seguidas das causais implícitas. Para o GC o tipo de narrativa de maior ocorrência foi a causal implícita seguida da intencional explícita.

Considerando apenas as narrativas produzidas a partir das histórias mecânicas (Tabela 1), os grupos também se diferenciaram quanto ao tipo de narrativa $(X 2=68,16 ;$ g.l. $=5 ; \mathrm{p}<0,001 *)$. Para o GP houve um predomínio das descritivas com conectivos, seguido das causais implícitas. Já para o GC o tipo de narrativa de maior ocorrência foi a causal implícita seguida da intencional explícita.

Analisando as histórias comportamentais (Tabela 2), os grupos também se diferenciaram quanto ao tipo de narrativa $(\mathrm{X} 2=39,994 ;$ g.l. $=5 ; \mathrm{p}$ $<0,001 *)$. Houve um predomínio das descritivas com conectivos, seguido das descritivas sem conectivos no GP. No GC, predominaram as causais implícitas seguidas das intencionais explícitas.

Nas histórias intencionais (Tabela 3) os grupos também se diferenciam $(\mathrm{X} 2=31,886$; g.l. $=4 ; \mathrm{p}<$ $0,001 *)$. As descritivas sem conectivo foram excluídas da comparação devido à baixa freqüência. Para o GP houve predomínio das descritivas com conectivos, seguido das causais implícitas. Já para o GC o tipo de narrativa de maior ocorrência foi a causal implícita seguida da intencional explícita.

Os grupos também se diferenciam quanto ao conteúdo das narrativas. Para o GC, 100\% delas apresentaram conteúdo pertinente, enquanto para o GP, $90 \%$ foram pertinentes $(\mathrm{X} 2=36,92$; g.l. $=1$; $\mathrm{p}<$ $0,001 *)$. Os conteúdos foram coerentes em $93,6 \%$ das narrativas do GC e em 51,7\% das do GP (X2 = 112,88; g.l.=1; p <0,001*). Em relação à completude, $90,3 \%$ do GC e $47,5 \%$ do GP completaram as narrativas $(\mathrm{X} 2=101,36 ; \mathrm{g} .1 .=1 ; \mathrm{p}<0,001 *)$.
TABELA 1. Distribuição do tipo de narrativa por grupo - Seqüências Mecânicas.

\begin{tabular}{|c|c|c|c|c|c|c|}
\hline $\begin{array}{l}\text { Tipo de } \\
\text { Narrativa }\end{array}$ & $\begin{array}{l}\text { Descritivo } \\
\text { Sem } \\
\text { Conectivo }\end{array}$ & $\begin{array}{l}\text { Descritivo } \\
\text { Com } \\
\text { Conectivo }\end{array}$ & $\begin{array}{l}\text { Causal } \\
\text { Implícito }\end{array}$ & $\begin{array}{l}\text { Causal } \\
\text { Explícito }\end{array}$ & $\begin{array}{l}\text { Intencional } \\
\text { Implícito }\end{array}$ & $\begin{array}{l}\text { Intencional } \\
\text { Explícito }\end{array}$ \\
\hline GC & 0 & $23(16 \%)$ & $\begin{array}{c}88 \\
(61 \%)\end{array}$ & $4(2,8 \%)$ & $5(3,5 \%)$ & $24(16,7 \%)$ \\
\hline GP & $\begin{array}{c}14 \\
(29,2 \%)\end{array}$ & $\begin{array}{c}19 \\
(39,6 \%)\end{array}$ & $\begin{array}{c}15 \\
(31,3 \%)\end{array}$ & 0 & 0 & 0 \\
\hline \multicolumn{7}{|c|}{$X^{2}=68,158 ;$ g.l. $=5 ; p<0,001 *$} \\
\hline
\end{tabular}

TABELA 2. Distribuição do tipo de narrativa por grupo - Seqüências Comportamentais

\begin{tabular}{c|c|c|c|c|c|c}
\hline $\begin{array}{c}\text { Tipo de } \\
\text { Narrativa }\end{array}$ & $\begin{array}{c}\text { Descritivo } \\
\text { sem } \\
\text { Conectivo }\end{array}$ & $\begin{array}{c}\text { Descritivo } \\
\text { com } \\
\text { Conectivo }\end{array}$ & $\begin{array}{c}\text { Causal } \\
\text { Implícito }\end{array}$ & $\begin{array}{c}\text { Causal } \\
\text { Explícito }\end{array}$ & $\begin{array}{c}\text { Intencional } \\
\text { Implícito }\end{array}$ & $\begin{array}{c}\text { Intencional } \\
\text { Explícito }\end{array}$ \\
\hline GC & 0 & $\begin{array}{c}29 \\
(20,1 \%)\end{array}$ & $\begin{array}{c}60 \\
(41,7 \%)\end{array}$ & $6(4,2 \%)$ & $14(9,7 \%)$ & $35(24,3 \%)$ \\
GP & $7(15,2 \%)$ & $\begin{array}{c}20 \\
(13,9 \%) \\
\end{array}$ & $13(9 \%)$ & 0 & $4(2,8 \%)$ & $2(1,4 \%)$ \\
$X^{2}=39,994 ;$ g.l. $=5 ; \mathrm{p}<0,001 *$ \\
\hline
\end{tabular}

TABELA 3. Distribuição do tipo de narrativa por grupo - Seqüências Intencionais.

\begin{tabular}{c|c|c|c|c|c|c}
\hline $\begin{array}{c}\text { Tipo de } \\
\text { Narrativa }\end{array}$ & $\begin{array}{c}\text { Descritivo } \\
\text { Sem } \\
\text { Conectivo }\end{array}$ & $\begin{array}{c}\text { Descritivo } \\
\text { Com } \\
\text { Conectivo }\end{array}$ & $\begin{array}{c}\text { Causal } \\
\text { Implícito }\end{array}$ & $\begin{array}{c}\text { Causal } \\
\text { Explícito }\end{array}$ & $\begin{array}{c}\text { Intencional } \\
\text { Implícito }\end{array}$ & $\begin{array}{c}\text { Intencional } \\
\text { Explícito }\end{array}$ \\
\hline GC & 0 & 0 & $\begin{array}{c}28 \\
(38,9 \%)\end{array}$ & $\begin{array}{c}8 \\
(11,1 \%)\end{array}$ & $9(12,5 \%)$ & $27(37,5 \%)$ \\
GP & 1 & $8(38,1 \%)$ & $\begin{array}{c}7 \\
(33,3 \%) \\
X^{2}=31,886 ; g .1 .=4 ; \mathrm{p}<0,001^{*}\end{array}$ & $2(9,5 \%)$ \\
\hline
\end{tabular}




\section{Discussão}

As crianças com DEL apresentam narrativas mais rudimentares se comparadas às crianças de mesma idade com desenvolvimento típico de linguagem, independente do tipo de história fornecida. Isto mostra que a dificuldade destas crianças está na utilização da língua, ou seja, nas habilidades lingüísticas necessárias na narração de histórias e não na percepção dos estados mentais dos personagens. Além disso, verificou-se que com relação à coesão e completude das histórias houve grande discrepância entre os grupos, mostrando a inabilidade dos sujeitos com DEL em lidar com estruturas morfossintáticas.

Estes dados corroboram outros estudos, em que crianças com DEL produziram histórias menores, com menos coesão e com muitos erros de sintaxe, semântica e morfologia devido aos déficits lingüísticos que caracterizam estes sujeitos ${ }^{(8,17-18)}$.

Newman e Macgregor (19) apontaram que estruturalmente, a narrativa produzida pelas crianças com DEL e crianças com desenvolvimento normal diferiram em diversos aspectos. As histórias produzidas por crianças com DEL foram menores, apresentaram extensão média do enunciado (EME) abaixo do esperado para a faixa etária, maior proporção de frases agramaticais e número significativamente menor de elementos do enredo da história se comparadas a seus pares cronológicos com desenvolvimento normal de linguagem.

Outras pesquisas mostraram que as narrativas produzidas por crianças com DEL são caracterizadas pelo menor número de componentes gramaticais na história, baixo número de episódios completos, e pouca coesão se compararmos ao desempenho observado em seus pares cronológicos com desenvolvimento normal de linguagem ${ }^{(17,20-23)}$. Além disso, comparando com seus pares, as crianças com DEL produzem um número significativamente menor de sentenças sintaticamente complexas, menos frases elaboradas e maior número de erros gramaticais ${ }^{(8,20,24-27)}$.

\section{Conclusão}

Os resultados deste estudo mostram que os grupos se diferiram quanto ao tipo de narrativa e conteúdo, de forma que as crianças com DEL apresentaram maior ocorrência de narrativa descritiva com conectivo enquanto as crianças em desenvolvimento normal de linguagem apresentaram maior número de narrativas causais implícitas. Além disso, houve diferença em relação ao conteúdo da narrativa, já que enquanto quase todas as narrativas produzidas pelas crianças do GC eram coerentes e completas, somente metade das produzidas pelo GC se configurou dessa forma.

Os déficits observados na produção narrativa em crianças com DEL apontam para as dificuldades conversacionais presentes nestes sujeitos que influenciam, dessa forma, no desenvolvimento da competência social, que implica em aspectos sociais, cognitivos, acadêmicos e comportamentais.

Finalizando, destaca-se a importância da realização da avaliação das habilidades narrativas durante o processo diagnóstico em linguagem sempre que possível, ou mesmo durante o processo terapêutico, já que permitem ao fonoaudiólogo verificar as competências lingüísticas, cognitivas e sociais destas crianças, além de fornecer dados importantes para o encaminhamento mais efetivo do processo terapêutico. 


\section{Referências Bibliográficas}

1. Stark RE, Tallal P. Selection of children with specific language deficits. Journal of Speech and Hearing Disorders. 1981;46:114-22.

2. Bishop DV, Adams C. Comprehension problems in children with specific language impairment: literal and inferential meaning. Journal of Speech and Hearing Disorders. 1992;35:119-29.

3. Crespo-Eguílaz N, Narbona J. Perfiles clínicos evolutivos y transiciones em el espectro del transtorno específico del desarrollo del lenguaje. Rev. Neurol. 2003;36(1):29-35.

4. Rocha LC, Befi-Lopes DM. Análise pragmática das respostas de crianças com e sem distúrbio específico de linguagem. Pró-Fono Rev. Atual. Cient. 2006;18:229-39.

5. Hage SRV, Joaquim RSS, Carvalho KG, Padovani CR, Guereiro MM . Diagnóstico de crianças com alterações específicas de linguagem por meio de escala de desenvolvimento. Arquivos de Neuro-Psiquiatria, São Paulo. 2004;62(3A):649-53

6. Reilly J, Losh M, Bellugi U, Beverly W. "Frog, Where Are You?" Narratives in Children with Specific Language Impairment, Early Focal Brain Injury, and Williams Syndrome. Brain and Language. 2004;88:229-47.

7. Botting N. Narrative as a clinical tool for the assessment of linguistic and pragmatic impairments. Child Language Teaching and Therapy. 2002;43:917-31.

8. Bishop DVM, Norbury CF. Narrative skills of children with communication impairments. Int. J. Lang. Comm. Dis. 2003;38:287-313.

9. Craig HK, Evans JL. Turn exchange characteristics of SLI children's simultaneous and nonsimultaneous speech. Journal of Speech and Hearing Disorders. 1989;54:334-47.

10. Befi-Lopes DM, Rodrigues A, Rocha LC. Habilidades lingüístico-pragmáticas em crianças normais e com alteração no desenvolvimento da linguagem. Pró-Fono Rev. Atual. Cient. 2004;16(1):57-66.

11. Westby C, Van Dongen R, \& Maggart Z. Assessing narrative competence. Seminars in Speech and Language. 1989;10:63-75.

12. Andrade CRF, Befi-Lopes DM, Fernandes FDM, Wertzner HF. Manual de avaliação de linguagem do serviço de Fonoaudiologia do centro de saúde escola Samuel B. Pessoa. Publicação Interna, 127 f. São Paulo, 1997.

13. Wertzner HF. Fonologia. In: Andrade CRF de, BefiLopes DM, Fernandes FDM, Wertzner HF. ABFW - Teste de linguagem infantil: nas áreas de fonologia, vocabulário, fluência e pragmática. Barueri: Pró-Fono; 2004. cap. 1.

14. Baron-Cohen S, Leslie AM, Frith U. Mechanical, Behavioural and Intencional understanding of stories in autistic children. British Journal of Developmental Psychology. 1986;4:113-25.
14. Perissinoto J. Avaliação Fonoaudiológica da criança com Autismo, IN Perissinoto, J. (Org) Conhecimentos Essenciais para atender bem a criança com Autismo. Ed Pulso; 2003. cap. 5. p. 45-55.

16. Perroni MC. O Desenvolvimento do Discurso Narrativo. Ed. Martins Fontes, São Paulo; 1992.

17. Merrit DD, Lilies BZ. Story grammar ability in children with and without language disorder: Story generation, story retelling, and story comprehension. Journal of Speech, Language, and Hearing Research. 1987;30:539-52.

18. Liles BZ, Duffy RJ, Merritt DD, Purcell SL. Measurement of narrative discourse ability in children with language disorders. Journal of Speech and Hearing Research. 1995;38:415-25

19. Newman RM, Mcgregor KK. Teachers and Laypersons discern quality differences between narratives produced by children with or without SLI. Journal of Speech, Language, and Hearing Research. 2006;49:1002-36.

20. Roth FP, Spekman NJ. Narrative discourse: Spontaneously generated stores of learning-disabled and normally achieving students. Journal of Speech, Language, and Hearing Research. 1986;51:8-23.

21. Gillam R, Mcfadden TU, Van Kleeck A. Improving narrative abilities: Whole language and language skills approaches. In Fey M, Windsor J, Warren SF. (Eds), Language intervention: Preschool through the elementary year, Baltimore: Brookes; 1995. p. 145-82.

22. Liles BZ. Cohesion in the narratives of normal and language-disordered children. Journal of Speech, Language, and Hearing Research. 1985a;28:123-33.

23. Liles BZ. Production and comprehension of narrative discourse in normal and language disordered children Journal of Communication Disorders. 1985b;18:409-27.

24. Maclachlan BG, Chapman RS. Communication breakdowns in normal and language learning disabled children's conversation and narration. Journal of Speech, Language, and Hearing Research. 1988;53:2-7.

25. Gillan R, Johnston JR. Spoken and written language relationship in language/learning-impaired and normally achieving school-age children. Journal of Speech, Language, and Hearing Research. 1992;35:1303-15.

26. Scott CM, Windsor J. General language performance measures in spoken and written narrative and expository discourse of school-age children with language learning disabilities. Journal of Speech, Language, and Hearing Research. 2000;43:324-39.

27. Greenhalgh, K.S.; Strong, C.J. Literate language features in spoken narratives of children with typical language and children with language impairments. Language, Speech, and Hearing Services in Schools, v.32, p.114-125, 2001. 